\title{
STUDIES IN DENERVATION
}

\section{F.-THE CIRCULATION IN THE SKIN OF THE PROXIMAL PARTS OF THE LIMBS}

BY

\author{
J. DOUPE, * C. H. CULLEN, AND L. J. MACAULAY
}

(From the E.M.S. Hospital, Winwick, nr. Warrington)

(RECEIVED 27TH OCTOBER, 1943)

THE influence of the vasomotor nerves on the circulation in the fingers and toes is readily demonstrable and has been extensively studied. It is, however, much more difficult to demonstrate the nervous regulation of the circulation in the skin of the proximal parts of the limbs and relatively few observations bearing on this aspect of the circulation have been recorded, while only Grant and Holling (1937-8) have made it the object of detailed study.

These authors found that very intense warming of the body produced an increased flow of blood in the skin of the limbs proximal to the hands and feet and by their method of occluding the circulation distal to the points under observation they were able to show that this increase was local and not merely a reflection of the changes occurring more peripherally. They then made experiments to elucidate the nature of this vasodilatation. They demonstrated that body warming failed to cause this vasodilatation after section of the sympathetic nerves or after cutting or blocking the local nerve supply of the skin. They next demonstrated that a local nerve block when the subject was cold did not cause a vasodilatation such as results in the digits and they made the complementary observation showing that a nerve block in the presence of vasodilatation caused a vasoconstriction. From these facts they concluded that the blood vessels of the skin of the proximal part of the limbs are supplied by vasodilator nerves of sympathetic origin.

In reporting their observations Grant and Holling left two points for future clarification. The first of these was in regard to the importance of the part played by the activity of the sweat glands in determining the vasodilatation, though it was considered that this was likely to be small because a temporal coincidence of sweating and vasodilatation was sometimes absent. The second question related to explanation of the vasodilatation which they observed in the skin of the proximal parts of the limbs for the first week following sympathectomy.

It is the purpose of this paper to record results

* Working on behalf of the Medical Research Council. substantiating those of Grant and Holling and to contribute to the solution of these two problems.

\section{Methods}

The circumstances under which the observations were made were essentially those described in paper A. The skin temperature was measured with thermocouples held in place by narrow strips of adhesive plaster $2 \mathrm{~cm}$. from the junction and this temperature was used as an index of the blood flow. The thermocouples and surrounding skin were covered with a layer of oil to minimize the effect of evaporation. To rule out changes in temperature consequent on variations in flow in the veins care was taken to locate the couples away from the venous channels and in each experiment the venous back flow distal to the couple was stopped for 10-15 minutes to prove that the proper precautions had been taken. The several instances in which blocking the venous back flow caused a fall in temperature have been excluded from consideration. The subjects are described in paper A.

\section{Results}

In Fig. 1 are shown the results obtained from subject S.S. with a cervical ganglionectomy of three months' duration and from subjects H.D. and N.T. with lesions of peripheral nerves. It will be seen that in response to body warming the normally innervated areas showed an increase in temperature that was not dependent on an increased return of blood from the hands or feet. In areas in which the skin had lost either its sympathetic supply or its total innervation there was no response to prolonged heating of the body. These results, which have been confirmed in three other experiments on subjects with peripheral nerve lesions, confirm the observation of Grant and Holling on a patient with a lesion of the external popliteal nerve.

Four experiments were made to determine the effect of blocking various nerves when the subjects were cool. The internal saphenous, the external popliteal, the internal and the external cutaneous nerves of the forearm were blocked with 5-10 c.c. of 2 per cent. procaine in 1-50,000 adrenaline. In confirmation of Woollard and Phillips (1932-3) and Grant and Holling these procedures produced no alteration in skin temperature despite a complete interruption of conduction in the nerves as shown by loss of appreciation of pin prick and light touch. 


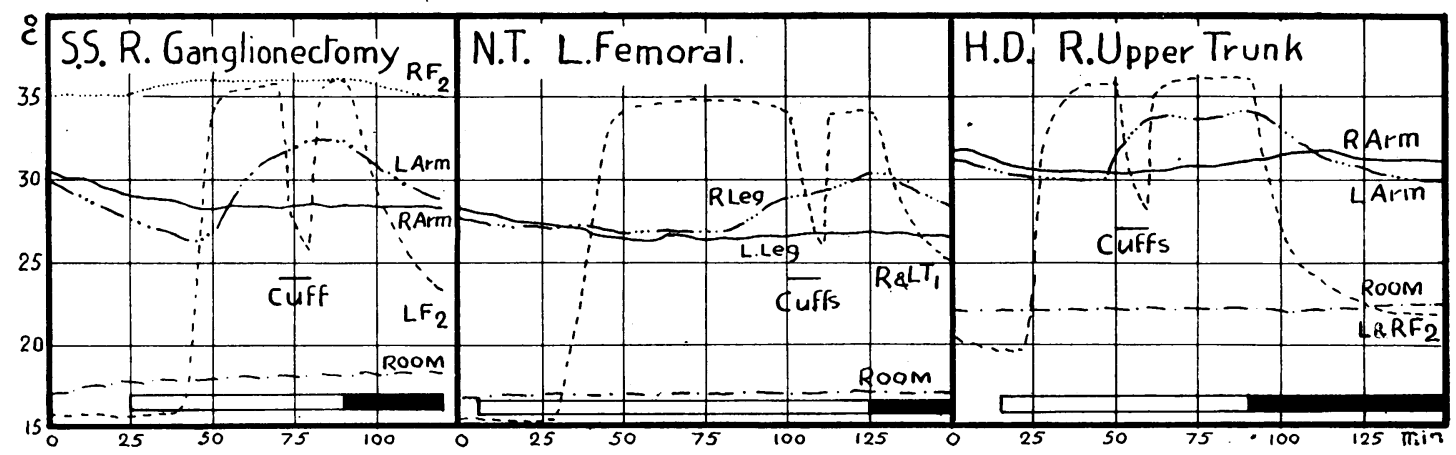

FIG. 1.-Temperature charts of various subjects, abscissæ are time in min. and ordinates are ${ }^{\circ} \mathrm{C}$. The temperature of the skin affected by the nerve lesion is represented by a continuous line while that of the normal contralateral area is represented by a line broken by three dots. The lines representing the temperature of the digits are indicated on the charts, F.2 indicates the index finger, while T.1 indicates the great toe. The blocks at the bottom of the charts show when empty that the indifferent limbs were immersed in water at $44^{\circ} \mathrm{C}$., when solid in water at $18-20^{\circ} \mathrm{C}$. The transverse line labelled cuff indicates the period during which the circulation of the hand or feet was occluded. In the cases of S.S. and H.D. the couples were on the lateral aspect of the upper arms while in that of N.T. they were on the medial aspect of the leg.

One experiment was made to determine the effect of local nerve block when the subject was warm. The result, which is shown in Fig. 2, indicates that vasoconstriction occurred following the nerve block.

These experiments confirm the results and deductions of Grant and Holling and it may therefore be confidently concluded that sympathetic fibres cause an active vasodilatation in the skin of the proximal parts of the limbs in response to intense body

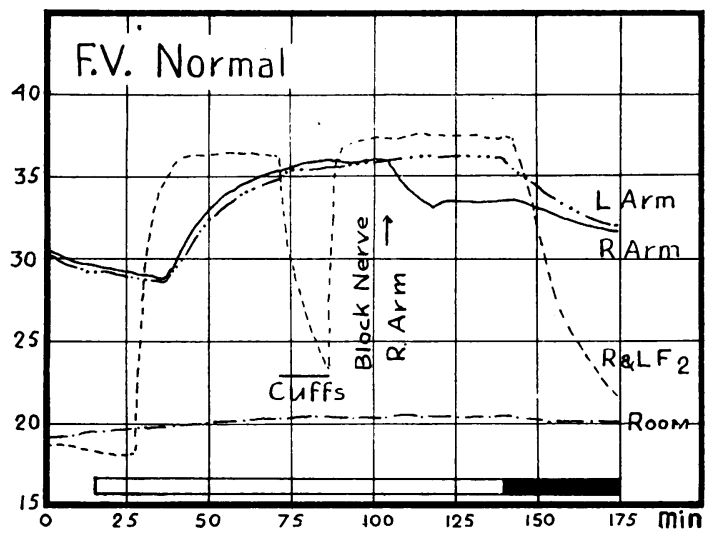

FIG. 2.-Temperature chart of a female volunteer, conventions as in Fig. 1 except that the continuous line represents the temperature of a point on the centre of the area supplied by the external cutaneous nerve of the forearm. This nerve was blocked by 5 c.c. 2 per cent. procaine in 1-50,000 adrenaline at the time indicated by the arrow. The temperature of three other points nearer the margins of this area showed a less definite fall in temperature following the nerve block.

heating. It may also be concluded from the lack of effect of nerve block when the subjects were cold that there is no significant vasoconstrictor innervation of the vessels of these areas of skin.

Nevertheless, Warren et al. (1942) found evidence that blocking the cervical sympathetic outflow did produce an increased blood flow in the proximal part of the limb, and while they did not distinguish between the cutaneous and the deep circulation they have used this as a basis for criticizing the whole concept of vasodilator nerves. As Barcroft et al. (1943) have demonstrated vasoconstrictor nerves to the muscles of the forearm it was necessary to repeat the experiment with particular reference to the skin. The right cervical sympathetic chain of a normal volunteer was blocked with 2 per cent. procaine and 1-50,000 adrenaline, and the results are shown in Fig. 3. It will be seen that the pro-

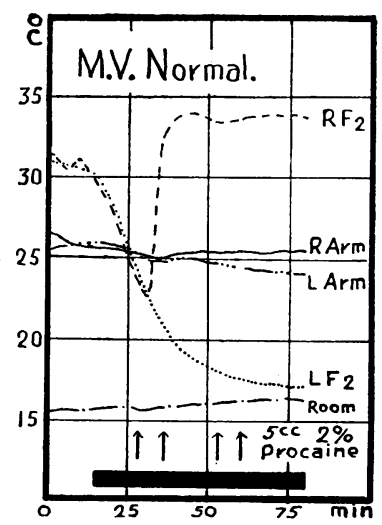

FIG. 3.-Temperature chart of a male volunteer. Conventions as in Fig. 1 except that the continuous line represents the temperature of an area on the lateral aspect of the upper arm. Arrows indicate the time at which 5 c.c. of 2 per cent. procaine in 1-50,000 adrenaline were injected into the region of the cervico-thoracic sympathetic trunk. A complete Horner's syndrome was not obtained until after the fourth injection.

cedure produced a paralysis of the vasoconstrictor fibres to the fingers, a definite Horner's syndrome was also present, but there was no change in the skin temperature of the arm. This result therefore confirms the deduction that there are no tonic neurogenic vasoconstrictor impulses to the vessels of the skin of the proximal part of the limbs.

SUDOMOTOR INFLUENCE ON THE VASODILATATION. -It has been noted that Grant and Holling did not to their entire satisfaction rule out the influence 
of the activity of sweat glands in the production of the vasodilatation. Carmichael (1943) suggested that this might be done by utilizing the paralysing action of atropine. The experiment, reported in Fig. 4, was therefore performed and it will be seen

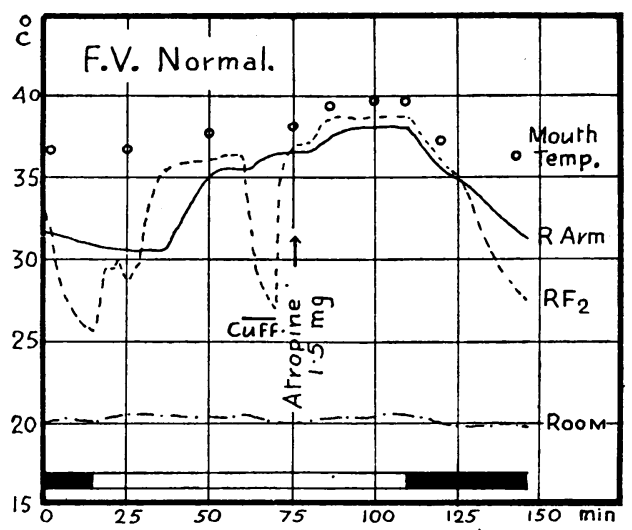

Fig. 4.- Temperature chart of a female volunteer, conventions as in Fig. 1 except that the continuous line represents the temperature of a normal area on the lateral aspect of the upper arm. Circles indicate mouth temperature. 1.5 mg. atropine was injected subcutaneously into the left arm at the time indicated by the arrow.

that the vasodilatation was not decreased, although there was a complete cessation of sweat production. The change in skin temperature was in fact indicative of a vasodilatation, but as it was proportional to the rise in the body temperature it may be concluded that no alteration actually occurred in the blood flow.

Atropine perhaps does not completely prevent all nervous activation of the sweat glands, but Barcroft (1901) has shown in the case of the salivary glands that it prevents the increase of $\mathrm{O}_{2}$ usage. It is reasonable to assume that the metabolism of the sweat glands would be similarly affected. Therefore it seems unlikely that the activation of the sweat glands determines the vasodilatation in question. Certain limitations to this conclusion are implied by the consideration that impulses in the sudomotor fibres may release a diffusible vasodilator substance belonging either to the choline or to some other group whose action on the blood vessels is not prevented by the amount of atropine given in this case.

Cause of Vasodilatation following SymPATHECTOMY.-Reference has been made to the finding of Grant and Holling in two cases of lumbar sympathectomy that the temperature of the skin of the legs was elevated for 5-6 days following operation. They were unable to reconcile this with their other results and discussed the two following possibilities.

1. A sympathectomy would have an effect on all the vessels of the limb, whereas a nerve block would only influence the terminal vessels. It is therefore possible that a vasoconstriction in the proximal vessels might prevent an increased blood flow following a block of a peripheral nerve. This possibility may be excluded because it has been found here that a procaine block of the sympathetic chain is similar to a block of a peripheral nerve in that neither induces a vasodilatation.

2. The products of nerve degeneration might cause vasodilatation and these would be absent in a nerve block, but present after a sympathectomy. This theory had certain attractions, for as Grant and Holling observe it would be a counterpart of the theory which Trotter and Davies (1909) evolved to explain the hyperæsthesia following nerve section. It was also attractive because it could be put to experimental test. Accordingly a branch of the long saphenous nerve was crushed in a volunteer. The area denervated is seen in Fig. 5 in which is

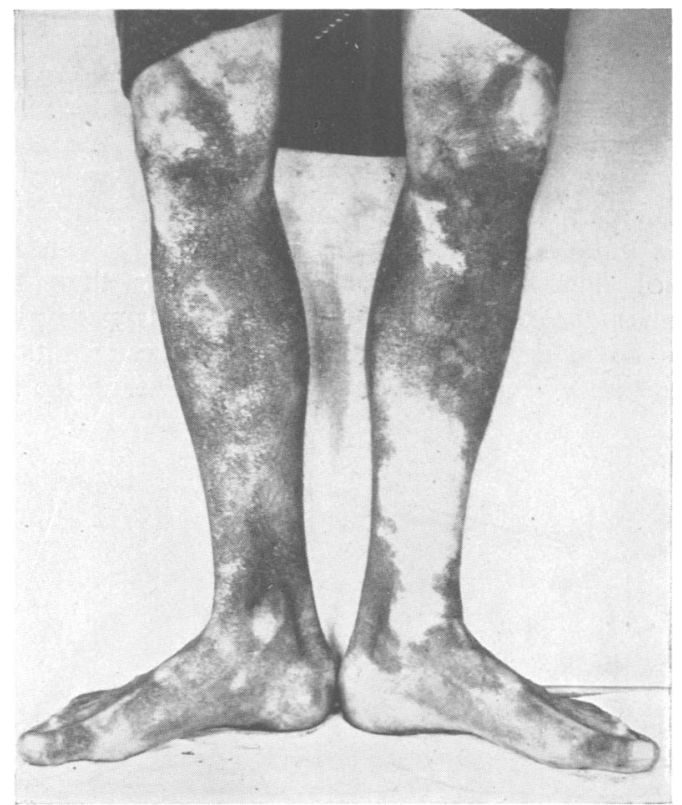

FIG. 5.-Photograph of the sweat response to body heating of a male volunteer six weeks after crushing a branch of the long saphenous nerve. The site of the incision is indicated by the area of anhydrosis just below the knee.

shown the sweat response to body heating 6 weeks after operation. The area of sensory loss was slightly greater than the area in which sweating was lost. The flare response tested with histamine was also absent. Temperatures were taken from 20 points on the leg daily for 1 week preceding and 2 weeks following operation and thereafter at irregular intervals. The leg was exposed for 20-30 mins. at room temperature of $20^{\circ} \mathrm{C}$. before each series of readings. No difference in temperature of the denervated and normal areas developed till the 5th day. At that time the denervated areas was found to be about $1^{\circ} \mathrm{C}$. cooler than the adjacent regions and this difference with minor variations persisted for 6 weeks when the observations were discontinued. It is apparent, therefore, that the degeneration of nerves is not necessarily associated with vasodilatation. 
Since the publication of Grant and Holling's paper Smithwick (1940) has shown that section of preganglionic sympathetic fibres causes the decentralized cells to initiate impulses for the succeeding 5-6 days. It is not improbable, therefore, that the vasodilatation found after sympathectomy was due to discharges in the vasodilator fibres which were decentralized by the operation of lumbar sympathectomy. Other factors such as the anæsthetic and absorption from traumatized tissues might also be involved in the causation of the vasodilatation. It will be seen, therefore, that this phenomenon need present no obstacle to the complete acceptance of the main thesis of Grant and Holling.

It is of interest to note that Trotter and Davies (1909) reported that vasodilatation follows section of a peripheral nerve supplying the skin of the proximal parts of the limb. It is possible that their observations resulted in part from a dependence on subjective methods of determining the temperature. The fallacy of this method was well demonstrated in the present instance, because when the subject tested the denervated areas with his cool fingers it felt distinctly warmer than the remainder of the leg. The cause of this was found to be a mistaken localization of the sensation, because when the fingers were warmer than the leg the denervated area seemed to be cooler than the remainder.

\section{Summary}

The views, of Grant and Holling concerning the vasomotor innervation of the skin of the proximal parts of the limb have been summarized.

Their experiments have been repeated and confirmed in detail and their conclusion that vasodilatation is brought about by stimulation of cutaneous sympathetic nerves has been substantiated.

Fresh evidence has been brought to show that the vasodilatation is not dependent on the activity of the sweat glands.

Evidence has also been produced to show that the vasodilatation following sympathectomy need present no obstacle to the acceptance of these conclusions.

\section{REFERENCES}

Barcroft, J. (1901). J. Physiol., 27, 31.

Barcroft, H., Bonnar, W. M., Edholm, O. G., and Effron, A. S. (1943). Ibid. 102, 21.

Carmichael, E. A: (1943). Personal Communication.

Grant, R. T., and Holling, H. E. (1937-8). Clin. Sci., 3, 273.

Smithwick, R. H. (1940). Arch. Surg., 40, 286.

Trotter, W., and Davies, H. M. (1909). J. Physiol., 38, 134.

Warren, J. V., Walter, C. W., Romano, J., and Stead, E. A. (1942). J. clin. Invest., 21, 665.

Woollard, H. H., and Phillips, R. (1932-33). J. Anat., 67,18 . 